\title{
HIPOCONTRATILIDADE DO ESÔFAGO EM PACIENTES COM DOENÇA DE CHAGAS E PACIENTES COM ACALÁSIA IDIOPÁTICA
}

\author{
Roberto Oliveira DANTAS *
}

\begin{abstract}
RESUMO - Em pacientes com esofagopatia chagásica e pacientes com acalásia, a amplitude das contrações esofágicas está diminuida. Como nem todas as deglutições são seguidas de contrações com baixa amplitude, avaliamos o número de contrações com amplitude abaixo de $30 \mathrm{~mm} \mathrm{Hg} \mathrm{em} 40$ voluntários assintomáticos, 99 pacientes com doença de Chagas e 14 pacientes com acalásia idiopática. Cada paciente ou voluntário realizou 10 deglutições de $5 \mathrm{~mL}$ de água e o registro das contrações foi feito a $5 \mathrm{~cm}, 10 \mathrm{~cm}$ e $15 \mathrm{~cm}$ proximal ao esfincter inferior do esôfago. Neste registro foi utilizado o método manométrico com perfusão contínua. O número de contrações com hipocontratilidade foi maior nos pacientes com doença de Chagas e pacientes com acalásia do que nos voluntários $(P<0,05)$. Pacientes chagásicos com trânsito lento no exame radiológico mas sem dilatação do esôfago tiveram mais contrações com baixa amplitude do que pacientes com exame radiológico normal $(P<0,01)$. O mesmo resultado se obteve quando da análise do número de indivíduos com três ou mais contrações com baixa amplitude $(P<0,01)$. Os pacientes chagásicos com disfagia tiveram mais contrações com hipocontratilidade do que aqueles sem disfagia $(P<0,05)$. Concluímos que na doença de Chagas e na acalásia idiopática ocorre maior número de contrações esofágicas com baixa amplitude do que em pessoas normais, o que deve contribuir para a sintomatologia dos pacientes.
\end{abstract}

DESCRITORES - Doença de Chagas. Acalásia. Esôfago. Deglutição.

\section{INTRODUÇÃO}

Distúrbio motor inespecífico do esôfago inclui um grupo de pacientes em que as alterações motoras do órgão não se enquadram nas categorias bem estabelecidas de anormalidades da motilidade ${ }^{(9,10,27)}$. Mais de $90 \%$ destes pacientes têm hipocontratilidade esofágica ${ }^{(19,25)}$, ou seja, as contrações desencadeadas pela deglutição têm amplitude baixa.
$\mathrm{Na}$ esofagopatia conseqüente à doença de Chagas e na acalásia idiopática ocorre diminuição da amplitude da contração esofágica ${ }^{(4,5,10,22,27)}$. No entanto, não são todas as deglutições que são seguidas de contrações de baixa amplitude ou mesmo ausência de contrações.

Não é conhecido em qual proporção ocorrem contrações de baixa amplitude, após a deglutição de água, na doença de Chagas. O objetivo deste trabalho foi estudar a freqüência de contrações

Professor Associado do Departamento de Clínica Médica da Faculdade de Medicina de Ribeirão Preto da Universidade de São Paulo. Endereço para correspondência: Dr. Roberto Oliveira Dantas - Departamento de Clínica Médica - Faculdade de Medicina de Ribeirão Preto - USP - 14049-900 Ribeirão Preto, SP. 
com amplitude abaixo de um limite considerado aceitável e a relação com o grau de comprometimento esofágico, em pacientes com doença de Chagas e pacientes com acalásia idiopática. Embora nestas duas condições outros distúrbios motores estejam presentes, como contrações síncronas e relaxamento parcial ou ausente do esfíncter inferior do esôfago ${ }^{(10,23)}, \mathrm{o}$ componente de hipocontratilidade, freqüente nos distúrbios motores inespecíficos, deve contribuir para as manifestações da esofagopatia.

\section{MATERIAL E MÉTODO}

Foram estudados 99 pacientes com exame sorológico positivo para doença de Chagas, 14 pacientes com acalásia idiopática e 40 voluntários assintomáticos.

Entre os pacientes com doença de Chagas, 61 tinham exame radiológico do esôfago normal e 38 tinham o exame anormal, caracterizado por trânsito lento do meio de contraste, retenção e contrações terciárias. Nenhum paciente tinha dilatação do esôfago. O exame radiológico foi realizado em posição ortostática. Exame endoscópico dos pacientes com exame radiológico anormal não revelou lesões associadas. Disfagia foi referida por 13 pacientes $(21 \%)$ com exame radiológico normal, e 27 (71\%) com exame anormal. Nenhum paciente havia sido submetido a tratamento. A idade dos pacientes variou de 19 a 78 anos, sendo que aqueles com exame radiológico normal tinham média \pm DP de idade de 42,9 $\pm 12,7$ anos e com exame anormal $51,5 \pm 11,1$ anos.

Os pacientes com acalásia idiopática foram investigados por apresentarem queixa de disfagia. Os exames endoscópico, radiológico e manométrico foram compatíveis com o diagnóstico ${ }^{(10,27)}$. Na manometria havia relaxamento parcial ou ausente do esfíncter inferior do esôfago e contrações síncronas. Retenção do meio de contraste foi observado no exame radiológico do esôfago. No exame endoscópico não havia lesões associadas. Todos tinham exame sorológico negativo para doença de Chagas e nenhum havia sido submetido a tratamento. Neste grupo de pacientes não havia dilatação do esôfago. A idade variou de 23 a 69 anos, com média de $44,5 \pm 14,0$ anos.

Voluntários assintomáticos formaram o grupo controle. Tinham idades entre 21 e 70 anos, média de 37,5 \pm 14,3 anos. Consentimento para a realização do exame manométrico foi obtido de cada paciente e voluntário, sendo a utilização da manometria do esôfago aprovada pela Comissão de Normas Éticas e Regulamentares do Hospital das Clínicas de Ribeirão Preto.

No exame manométrico foi utilizada uma sonda de polivinil de oito canais com 4,5 mm de diâmetro externo e $0,8 \mathrm{~mm}$ de diâmetro interno em cada canal (Arndorfer Specialties Inc, Greendale, Wisconsin, USA). As aberturas laterais dos quatro canais proximais distanciavam-se $5 \mathrm{~cm}$, formando ângulos de $90^{\circ}$. Cada canal foi conectado a transdutores de pressão (Modelo RP 1500, Narco Bio Systems, Narco Scientific, Houston, Texas, USA) com saídas para um fisiógrafo de quatro canais (Modelo MK IV, Narco Bio Systems, Narco Scientific). Cada canal foi perfundido continuamente com água destilada a um fluxo de $0,5 \mathrm{~mL} / \mathrm{min}$ através de um sistema de perfusão de baixa complacência.

A metodologia de realização de manometria do esôfago foi previamente descrita ${ }^{(3,}$ ${ }^{6,11)}$. Todos os sujeitos foram estudados em posição supina. A sonda foi introduzida pela narina até o estômago após jejum de 12 horas. Para registrar as contrações, o canal mais distal dentre os quatro proximais foi localizado no esfíncter inferior do esôfago e os outros a $5 \mathrm{~cm}, 10 \mathrm{~cm}$ e $15 \mathrm{~cm}$ acima do esfíncter. Nesta posição, voluntários e pacientes realizaram 10 deglutições de $5 \mathrm{~mL}$ de água destilada na temperatura ambiente alternadas com 10 deglutições "secas", com um intervalo mínimo de 30 segundos entre elas. Para análise da amplitude das contrações consideramos apenas as deglutições de água. O exame manométrico compreendia também as medidas das pressões dos esfíncteres inferior e superior do esôfago ${ }^{(3,11)}$. A pressão do esfíncter inferior foi medida pelo método da retirada rápida $^{(3,6)}$. As contrações no corpo do esôfago foram consideradas síncronas quando tinham o mesmo início nos canais localizados a 10 e 5 $\mathrm{cm}$ acima do esfíncter inferior. O relaxamento do esfíncter foi considerado completo quando sua pressão caia a menos de $4 \mathrm{~mm} \mathrm{Hg}$ acima da pressão intragástrica, após a deglutição de 5 $\mathrm{mL}$ de água. Ausência de relaxamento ocorreu quando a pressão do esfíncter não se modificava com a deglutição, sendo ele parcial quando havia diminuição da pressão do esfíncter porém a valores superiores a $4 \mathrm{~mm}$ Hg acima da pressão intragástrica ${ }^{(3,6)}$.

A amplitude da contração foi medida a partir da pressão intra-esofágica, quando dos intervalos entre as deglutições e com o sujeito respirando normalmente, até o pico da onda de contração (Figura 1). A amplitude foi considerada baixa (hipocontratilidade) quando seu valor foi inferior a $30 \mathrm{~mm} \mathrm{Hg}$ ou quando havia ausência de contração em determinado local do esôfago. Referência à hipocontratilidade distal foi feita quando a amplitude das contrações medidas $5 \mathrm{~cm}$ acima do esfíncter inferior foi menor do que $30 \mathrm{~mm} \mathrm{Hg}$ em três ou mais deglutições. O valor de 30 $\mathrm{mm} \mathrm{Hg}$ foi escolhido em função de resultados de trabalhos presentes na literatura ${ }^{(17,19)}$ e de voluntários assintomáticos ${ }^{(3,4,6)}$, onde a média da amplitude após 10 deglutições de água foi superior a $30 \mathrm{~mm} \mathrm{Hg}$ em todos.

Em cada voluntário e paciente contamos o número de deglutições seguidas de contrações com amplitude abaixo de $30 \mathrm{~mm} \mathrm{Hg}$ ou ausente, em cada local do esôfago. Os resultados são apresentados como a média \pm DP e mediana do número de deglutições com hipocontratilidade, e o número de sujeitos com três ou mais deglutições com hipocontrati- 


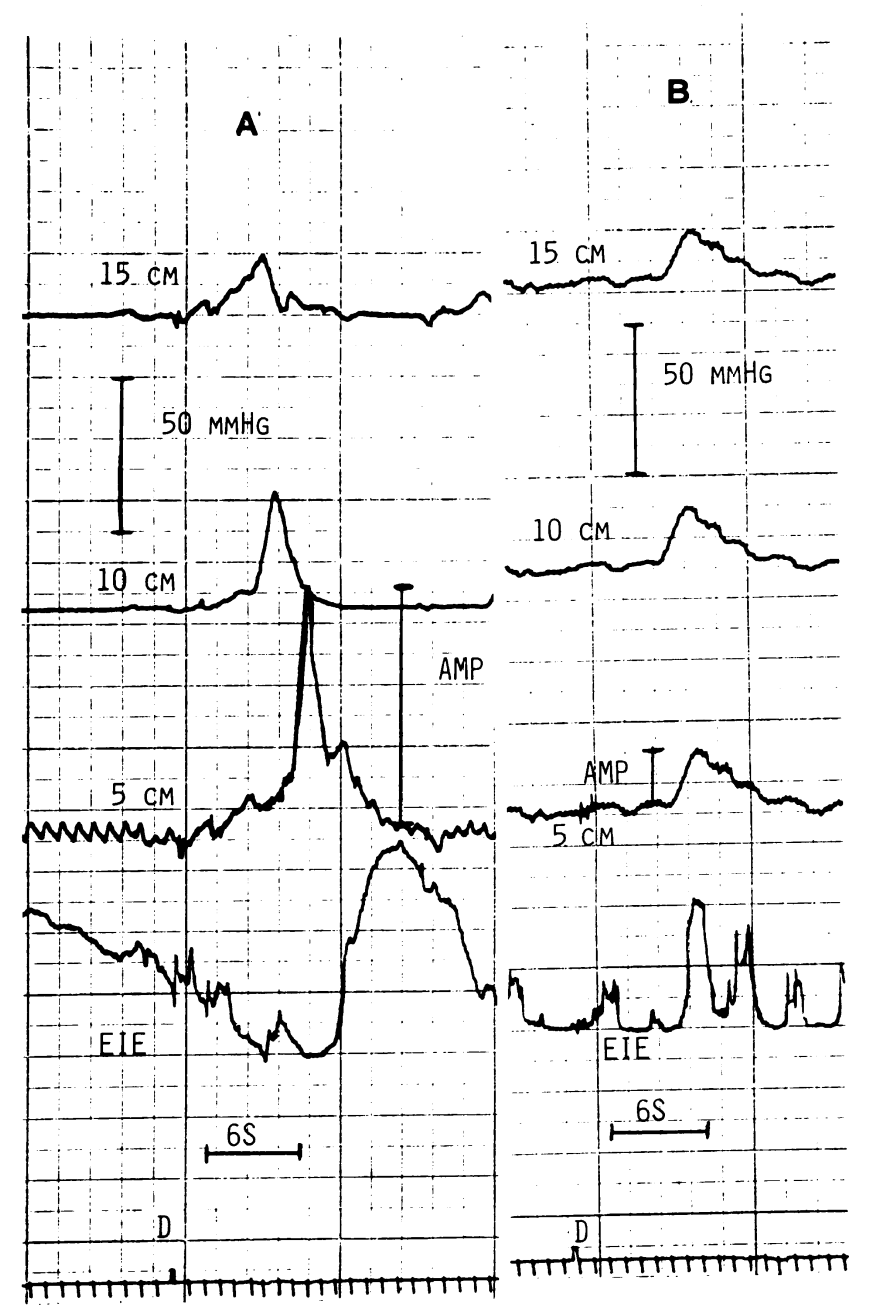

Figura 1 - A. Exame manométrico do esôfago de voluntário normal, com hipocontratilidade $15 \mathrm{~cm}$ acima do esfíncter inferior do esôfago (EIE). B. Paciente com acalásia e hipocontratilidade a $5 \mathrm{~cm}, 10 \mathrm{~cm}$ e $15 \mathrm{~cm}$ acima do EIE D - Deglutição de $5 \mathrm{~mL}$ de água. AMP - amplitude. S - segundos.

lidade $^{(19,25)}$. Para análise estatística dos resultados, utilizamos os testes de KruskalWallis, de Dunn para comparações múltiplas, qui-quadrado e t de Student.

\section{RESULTADOS}

Os resultados da média e mediana do número de deglutições seguidas de hipocontratilidade estão apresentados na Tabela 1. A ocorrência de hipocontratilidade no grupo de voluntários assintomáticos aconteceu em pequeno número de deglutições, principalmente em parte distal onde nenhum indivíduo teve mais de três contrações com baixa amplitude.

O número de contrações com hipocontratilidade foi maior nos pacientes com doença de Chagas e pacientes com acalásia do que nos voluntários assintomáticos $(P<0,05)$. Os pacientes chagásicos com exame radiológico do esôfago anormal tiveram maior número de contrações de baixa amplitude do que os pacientes chagásicos com exame radiológico normal $(P<0,01)$. Não houve diferenças entre os pacientes com doença de Chagas e pacientes com acalásia $(P>0,05)$.
O número de indivíduos com três ou mais deglutições, seguidas de contrações com baixa amplitude apresentou a mesma tendência (Tabela 2). Em parte distal do esôfago somente um voluntário apresentou três deglutições com contrações de baixa amplitude. O número de indivíduos foi maior entre os pacientes com doença de Chagas e pacientes com acalásia do que nos voluntários normais, e maior nos chagásicos com exame radiológico do esôfago anormal, do que naqueles com exame normal. Aumento do número de indivíduos com deglutições seguidas de hipocontratilidade ocorreu na seqüência de voluntários assintomáticos, pacientes com doença de Chagas e exame radiológico do esôfago normal, pacientes com acalásia e pacientes com doença de Chagas e exame radiológico anormal $(P<0,001)$.

Entre os pacientes chagásicos sem disfagia $(n=59)$, o percentual de contrações com amplitude baixa ou falha foi de $35 \%$, $26 \%$ e $27 \%$ a $15 \mathrm{~cm}, 10 \mathrm{~cm}$ e $5 \mathrm{~cm}$ do esfíncter inferior do esôfago, respectivamente. Nos pacientes com disfagia $(n=40)$, este percentual foi de $57 \%$, 49\% e $41 \%$ nos mesmos locais $(P<0,05)$. Quando comparados, somente os pacientes com exame radiológico normal, aqueles sem disfagia tiveram menor proporção de contrações com hipocontratilidade $(30 \%, 21 \%$ e $24 \%, \mathrm{n}=48)$ do que aqueles com disfagia $(38 \%, 34 \%$ e $22 \%, n=$ 13), a $15 \mathrm{~cm}$ e $10 \mathrm{~cm}$ do esfíncter inferior do esôfago $(P<0,05)$. Nos pacientes com exame radiológico anormal, aqueles sem disfagia (n $=11$ ) tiveram $60 \%, 48 \%$ e $36 \%$ de contrações com baixa amplitude, enquanto aqueles com disfagia $(\mathrm{n}=27)$ tiveram $66 \%, 56 \%$ e $49 \%$ de contrações com baixa amplitude $(P<0,05)$. A pressão do esfíncter inferior do esôfago foi de 23, $5 \pm 12,4 \mathrm{~mm} \mathrm{Hg}$ entre aqueles com hipocontratilidade distal $(\mathrm{n}=40)$ e de 27,0 \pm 13,3 mm Hg naqueles sem hipocontratilidade ( $\mathrm{n}=59, P>0,05)$. A relação entre relaxamento do esfíncter inferior e número de contrações síncronas com a contratilidade e disfagia está apresentada na Tabela 3. A proporção de 
Tabela 1 - Média e mediana do número de deglutições seguidas de hipocontratilidade após 10 deglutições de água, medida a $5 \mathrm{~cm}, 10 \mathrm{~cm}$ e 15 $\mathrm{cm}$ proximal ao esfíncter inferior do esôfago

\begin{tabular}{|c|c|c|c|c|c|c|c|c|c|}
\hline & \multicolumn{3}{|c|}{$15 \mathrm{~cm}$} & \multicolumn{3}{|c|}{$10 \mathrm{~cm}$} & \multicolumn{3}{|c|}{$5 \mathrm{~cm}$} \\
\hline & Média \pm DP & Mediana & Limites & Média \pm DP & Mediana & Limites & Média \pm DP & Mediana & Limites \\
\hline Voluntários & $1,2 \pm 1,9$ & 0 & $0-9$ & $0,9 \pm 1,7$ & 0 & $0-8$ & $0,4 \pm 0,7$ & 0 & $0-3$ \\
\hline \multicolumn{10}{|l|}{ Doença de Chagas } \\
\hline Radiologia normal & $3,1 \pm 3,3$ & $3 *+$ & $0-10$ & $2,3 \pm 3,0$ & $1+$ & $0-10$ & $2,3 \pm 3,3$ & $1^{*+}$ & $0-10$ \\
\hline $\begin{array}{l}\text { Radiologia anormal } \\
\text { Acalásia idiopática }\end{array}$ & $6,4 \pm 3,5$ & $7 * *$ & $0-10$ & $5,4 \pm 4,1$ & $6 * *$ & $0-10$ & $4,6 \pm 3,7$ & $4 * *$ & $0-10$ \\
\hline Radiologia anormal & $4,0 \pm 3,4$ & $3,5^{*}$ & $0-10$ & $5,3 \pm 4,5$ & $5,5^{*}$ & $0-10$ & $3,4 \pm 3,1$ & $3 *$ & $0-9$ \\
\hline
\end{tabular}

Tabela 2 - Número de voluntários e pacientes com três ou mais deglutições seguidas de hipocontratilidade, após 10 deglutições de água, medida a $5 \mathrm{~cm}, 10 \mathrm{~cm}$ e $15 \mathrm{~cm}$ proximal ao esfíncter inferior do esôfago

\begin{tabular}{|c|c|c|c|c|c|c|}
\hline & \multicolumn{2}{|c|}{$15 \mathrm{~cm}$} & \multicolumn{2}{|c|}{$10 \mathrm{~cm}$} & \multicolumn{2}{|c|}{$5 \mathrm{~cm}$} \\
\hline & $\mathbf{n}$ & $\%$ & n & $\%$ & $\mathbf{n}$ & $\%$ \\
\hline Voluntários & $7 *$ & 17,5 & $4^{*}$ & 10,0 & $1 *$ & 2,5 \\
\hline \multicolumn{7}{|l|}{ Doença de Chagas } \\
\hline Radiologia normal & 30 & 49,2 & 18 & 29,5 & 16 & 26,2 \\
\hline Radiologia anormal & 30 & 78,9 & 25 & 65,8 & 24 & 63,2 \\
\hline \multicolumn{7}{|l|}{ Acalásia idiopática } \\
\hline Radiologia anormal & 8 & 57,1 & 8 & 57,1 & 7 & 50,0 \\
\hline
\end{tabular}

TABELA 3 - Relaxamento do esfíncter inferior do esôfago (EIE) e número de contrações síncronas, em pacientes com doença de Chagas, relacionados ao número de pacientes com hipocontratilidade distal e disfagia

\begin{tabular}{|c|c|c|c|c|c|c|c|c|}
\hline \multirow[b]{3}{*}{ Relaxamento EIE } & \multicolumn{4}{|c|}{ Com hipocontratilidade } & \multicolumn{4}{|c|}{ Sem hipocontratilidade } \\
\hline & \multicolumn{2}{|c|}{$\begin{array}{l}\text { Com disfagia } \\
\quad(N=22)\end{array}$} & \multicolumn{2}{|c|}{$\begin{array}{l}\text { Sem disfagia } \\
\quad(N=18)\end{array}$} & \multicolumn{2}{|c|}{$\begin{array}{c}\text { Com disfagia } \\
\quad(\mathrm{N}=18)\end{array}$} & \multicolumn{2}{|c|}{$\begin{array}{l}\text { Sem disfagia } \\
\quad(n=41)\end{array}$} \\
\hline & $\mathrm{n}$ & $\%$ & $\mathrm{n}$ & $\%$ & $\mathrm{n}$ & $\%$ & $\mathrm{n}$ & $\%$ \\
\hline Completo & 8 & 40 & 12 & 60 & 10 & 23 & 34 & 77 \\
\hline Parcial & 9 & 64 & 5 & 36 & 7 & 54 & 6 & 46 \\
\hline Ausente & 5 & 83 & 1 & 17 & 1 & 50 & 1 & 50 \\
\hline \multicolumn{9}{|c|}{ Contrações síncronas } \\
\hline 0 & 2 & 17 & 10 & 83 & 13 & 29 & 32 & 71 \\
\hline $1-3$ & 6 & 55 & 5 & 45 & 2 & 22 & 7 & 78 \\
\hline $4-6$ & 4 & 67 & 2 & 33 & 1 & 50 & 1 & 50 \\
\hline $7-9$ & 0 & 0 & 1 & 100 & 0 & 0 & 1 & 100 \\
\hline 10 & 10 & 100 & 0 & 0 & 2 & 100 & 0 & 0 \\
\hline
\end{tabular}

$\mathrm{n}=$ número 
pacientes com relaxamento completo do esfíncter inferior e disfagia, foi maior naqueles com hipocontratilidade (40\%) do que naqueles sem hipocontratilidade (23\%). Também entre aqueles com até três contrações síncronas e disfagia, a proporção daqueles com hipocontratilidade (35\%) foi maior do que naqueles sem hipocontratilidade (28\%).

\section{DISCUSSÃO}

Os resultados indicam que pacientes com doença de Chagas e pacientes com acalásia têm maior número de contrações com baixa amplitude do que pessoas normais. Os pacientes com doença de Chagas e comprometimento radiológico do esôfago têm mais deglutições, seguidas de hipocontratilidade do que aqueles sem comprometimento radiológico. Os pacientes com acalásia não foram diferentes dos chagásicos. Em relação ao número de pacientes com três ou mais deglutições seguidas de hipocontratilidade, o número foi maior na doença de Chagas e na acalásia do que entre os voluntários, sendo que o número de pacientes com acalásia ficou entre os de chagásicos sem e com comprometimento radiológico do esôfago. Hipocontratilidade foi mais freqüente nos pacientes com disfagia do que naqueles sem disfagia.

A destruição do plexo mientérico do esôfago na doença de Chagas ${ }^{(18)}$ e na acalásia idiopática ${ }^{(12)}$, tem como conseqüência alterações na amplitude e a coordenação ${ }^{(10,22)}$ das contrações, semelhante ao que é obtido com o bloqueio de inervação colinérgica com atropina $^{(1,7)}$. Estudos em humanos observaram que o bloqueio da síntese de óxido nítrico (NO) aumenta a amplitude ${ }^{(14,21)}$. Na acalásia $^{(20)}$ e na esofagopatia chagásica ${ }^{(24)}$ a síntese de NO, que deveria estar presente na inervação inibitória não-adrenérgica nãocolinérgica, está diminuída. A diminuição da amplitude nos pacientes deve ser conseqüente à destruição de inervação colinérgica excitatória. A manutenção desta inervação com prejuízo de inervação inibitória provocaria aumento da amplitude. Associada à hipocontratilidade, os pacientes com acalásia ${ }^{(13)}$ e com doença de Chagas $^{(8)}$ têm complacência da musculatura do esôfago aumentada, o que significa menor aumento da tensão quando da distensão da parede do órgão.

A amplitude da contração acima de um valor mínimo é fundamental para a passagem de material deglutido, ou refluído, do esôfago para o estômago. Este valor em esôfago distal é de $30 \mathrm{~mm} \mathrm{Hg}^{(17)}$. Contrações de baixa amplitude são observadas em alguns pacientes com esofagite péptica ${ }^{(17)}$, sendo que quanto maior o comprometimento das contrações, mais graves as conseqüências do refluxo gastroesofágico $^{(16)}$. Com as doenças que causam prejuízo à inervação do esôfago, a alteração da motilidade é mais intensa, havendo alterações da amplitude e coordenação das contrações.

Estas contrações de baixa amplitude contribuem para a disfagia referida por estes pacientes e pela retenção do material deglutido em esôfago. Os resultados não indicaram diferenças na pressão do esfíncter inferior do esôfago relacionada à contratilidade, entretanto, sugerem que hipocontratilidade se relaciona com disfagia em pacientes com poucas contrações síncronas e relaxamento completo do esfíncter inferior do esôfago. Os mecanismos mais importantes para a retenção e a disfagia são as contrações síncronas no corpo do esôfago e o relaxamento parcial ou ausente do esfíncter inferior $^{(10,23,26,27)}$, todavia a baixa amplitude pode estar associada a sintomas como disfagia, pirose e dor torácica não-cardíaca ${ }^{(25)}$. É descrito que disfagia é referida por $19 \%{ }^{(25)}$ a $26 \%{ }^{(19)}$ dos pacientes com baixa amplitude de contração no esôfago. A hipocontratilidade permite que, quando ocorre refluxo gastroesofágico, o tempo de exposição da mucosa do esôfago ao material refluído seja maior ${ }^{(19,25)}$.

Os resultados sugerem que os pacientes com acalásia teriam menor número de contrações com hipocontratilidade, do que os pacientes com esofagopatia pela doença de Chagas, o que seria conseqüência de possível preservação parcial da inervação colinérgica excitatória no corpo do esôfago na acalásia, semelhante ao que acontece no esfíncter inferior $^{(15)}$ e contrário ao que acontece na doença de Chagas ${ }^{(2)}$, hipótese que necessita melhores estudos.

Em conclusão, na doença de Chagas e na acalásia idiopática é observada maior freqüência de contrações com amplitude diminuída, o que deve contribuir para a sintomatologia dos pacientes. 
Dantas RO. Esophageal hypotensive contractions in patients with Chagas' disease and patients with primary achalasia. Arq Gastroenterol 2000;37(1):35-41.

ABSTRACT - The esophageal contraction amplitude is low in patients with Chagas' disease and patients with primary achalasia but not every swallow is followed by low contraction amplitude. We evaluated the number of low contraction amplitude in 40 normal volunteers, 99 Chagas' disease patients and 14 patients with primary achalasia. Each subject performed 10 swallows of a $5 \mathrm{~mL}$ bolus of water and the esophageal motility was measured at 5,10 and $15 \mathrm{~cm}$ above the lower esophageal sphincter by the manometric method with continuous perfusion. The amplitude of contraction was considered to be low when its value was below $30 \mathrm{~mm} \mathrm{Hg}$. There was a hypotensive contraction when the amplitude was low or when the contraction failed. The number of hypotensive contractions was higher in patients with Chagas' disease and patients with achalasia than in healthy volunteers $(\mathrm{P}<0.05)$. Patients with Chagas' disease and abnormal esophageal radiological examination but without dilation had more frequent hypotensive contraction than patients with normal esophageal radiologic examination $(\mathrm{P}<0.01)$. The same results were obtained for subjects with three or more hypotensive contractions $(\mathrm{P}<0.01)$. The patients with Chagas' disease and dysphagia had more hypotensive contractions than patients without dysphagia (P $<0.05)$. We conclude that patients with Chagas' disease and patients with primary achalasia have a higher number of hypotensive contractions following wet swallows than normal volunteers, a fact that should influence the symptomatology of the patients.

HEADINGS - Chagas' disease. Achalasia. Esophagus. Swallowing.

\section{REFERÊNCIAS BIBLIOGRÁFICAS}

1. Conklin JL, Christensen J. Motor functions of the pharynx and esophagus. In: Johnson LR, ed. Physiology of the digestive tract. 3.ed. New York: Raven Press; 1994. p.903-8.

2. Dantas RO. Idiopathic achalasia and chagasic megaesophagus. J Clin Gastroenterol 1988;10:13.

3. Dantas RO, Ferriolli E, Souza MAN. Gender effects on esophageal motility. Braz J Med Biol Res 1998;31:539.

4. Dantas RO. Dysphagia in patients with Chagas' disease. Dysphagia 1998;13:53.

5. Dantas RO, Deghaide NHS, Donadi EA. Esophageal motility of patients with different grades of esophageal involvement by Chagas' disease. Gastroenterology 1998;114:A740. [Abstract].

6. Dantas RO, Aguiar LN, Ramos FS, Mamede RCM, Silva AK, Gonçalves AS, Mello Filho FV. Evaluation of esophageal motility in laryngectomized patients. Arq Gastroenterol 1999;36:112.

7. Dodds WJ, Dent J, Hogan WJ, Arndorfer RC. Effect of atropine on esophageal motor function in humans. Am J Physiol 1981;240:G290.

8. Ejima FH, Dantas RO, Simões MV, Marin Neto JA, Meneghelli UG. Intraesophageal ballon distension test in Chagas' disease patients with noncardiac chest pain. Dig Dis Sci 1998;43:2567.
9. Ergun GA, Kahrilas PJ. Clinical applications of esophageal manometry and ph monitoring. Am J Gastroenterol 1996;91:1077.

10. Feldman M. Esophageal achalasia syndromes. Am J Med Sci 1988;295:60.

11. Ferriolli E, Oliveira RB, Matsuda NM, Braga FJHN, Dantas RO. Aging, esophageal motility, and gastroesophageal reflux. J Am Geriatr Soc 1998;46:1534.

12. Goldblum JR, Whyte RI, Orringer MB, Alppelman HD. Achalasia. A morphologic study of 42 resected specimens. Am J Surg Pathol 1994;18:327.

13. Gonzales M, Mearin F, Vasconez C, Armengol JR, Malagelada JR. Oesophageal tone in patients with achalasia. Gut 1997;41:291.

14. Hirsch DP, Holloway RH, Tytgat GNJ, Boeckxstaens GEE. Involvement of nitric oxide in human transient lower esophageal sphincter relaxations and esophageal primary peristalsis. Gastroenterology 1998;115:1374.

15. Holloway RH, Dodds WJ, Helm JF, Hogan WJ, Dent J, Arndorfer RC. Integrity of cholinergic innervation to the lower esophageal sphincter in achalasia. Gastroenterology 1986;90:924.

16. Kahrilas PJ, Woods WJ, Hogan WJ, Kern M, Arndorfer RC, Reece A. Esophageal peristaltic dysfunction in peptic esophagitis. Gastroenterology 1986;91:897. 
17. Kahrilas PJ, Dodds WJ, Hogan WJ. Effect of peristaltilc dysfunction on esophageal volume clearance. Gastroenterology 1988;94:73.

18. Köberle F. Chagas' disease and Chagas' syndromes: The pathology of American trypanosomiasis. Adv Parasitol 1968;6:63.

19. Leite LP, Johnston BT, Barrett J, Castell JA, Castell DO. Ineffective esophageal motility (IEM). The primary findings in patients with nonespecific esophageal motility disorder. Dig Dis Sci 1997;42:1859.

20. Mearin F, Mourelle M, Guarner F, Salas A, Rivers-Moreno V, Moncada S, Malagelada JR. Patients with achalasia lack nitric oxide synthase in the gastro-oesophageal junction. Eur J Clin Invest $1993 ; 23: 724$

21. Murray JA, Ledlow A, Launspach J, Evans D, Loveday M, Conklin JL. The effects of recombinant human hemoglobin on esophageal motor function in humans. Gastroenterology 1995;109:1241.

22. Oliveira RB, Rezende Filho J, Dantas RO, Iazigi N. The spectrum of esophageal motor disorders in Chagas' disease. Am J Gastroenterol 1995;90:1119.
23. Oliveira RB, Troncon LEA, Dantas RO, Meneghelli UG. Gastrointestinal manifestations of Chagas' disease. Am J Gastroenterol 1998;93:884.

24. Oliveira RB, Matsuda NM, Antoniolli AR, Figueiredo FC, Ballejo G. Pharmacological and histological examination of intrinsic nerves in the esophagogastric region from chagasic patients with megaesophagus. Dig Dis Sci. [no prelo].

25. Pantoja JAS. Distúrbios motores inespecíficos em pacientes com sintomas esofagianos. Rio de Janeiro, 1998. [Dissertação de Mestrado - Faculdade de Medicina da Universidade Federal do Rio de Janeiro].

26. Rezende JM. The digestive tract in Chagas' disease. Mem Inst Oswaldo Cruz 1984;79 (Suppl):97.

27. Wong RKH, Maydonovitch CL. Achalasia. In: Castell DO, ed. The esophagus. 2.ed. Boston: Little, Brown; 1995. p.219-45.

Recebido para publicação em 13/5/1999. Aprovado para publicação em 31/8/1999. 